\title{
Derive Lovelock gravity from string theory in cosmological background
}

\author{
Peng Wang, ${ }^{a}$ Houwen Wu, ${ }^{a}$ Haitang Yang $^{a}$ and Shuxuan Ying $^{b}$ \\ ${ }^{a}$ College of Physics, Sichuan University, \\ 29 Wangjiang Road, Chengdu, 610065, China \\ ${ }^{b}$ Department of Physics, Chongqing University, \\ 55 South University Town Road, Chongqing, 401331, China \\ E-mail: pengw@scu.edu.cn, iverwu@scu.edu.cn, hyanga@scu.edu.cn, \\ ysxuan@cqu.edu.cn
}

ABstRACT: It was proved more than three decades ago, that the first order $\alpha^{\prime}$ correction of string effective theory could be written as the Gauss-Bonnet term, which is the quadratic term of Lovelock gravity. In cosmological background, with an appropriate field redefinition, we reorganize the infinite $\alpha^{\prime}$ corrections of string effective action into a finite term expression for any specific dimension. This finite term expression matches Lovelock gravity exactly and thus fix the couplings of Lovelock gravity by the coefficients of string effective action. This result thus provides a strong support to string theory.

Keywords: Bosonic Strings, String Duality, Classical Theories of Gravity

ARXIV EPRINT: 2012.13312 
Physically, an extension of general relativity requires the generalized Einstein tensor to respect three conditions: 1) It is symmetric; 2) It is divergence free (Bianchi identity); 3) Higher derivatives of the metric are absent in the EOM (ghost free). Lovelock gravity [1] is the most general metric theory extension satisfying these conditions. In $D=d+1$ dimensional spacetime, it is constructed by dimensionally extended Euler densities (setting $\left.16 \pi G_{D}=c=1\right)$ :

$$
\begin{aligned}
I_{\text {Love }} & =\int d^{D} x \sqrt{-g} \sum_{k=0}^{\left[\frac{D-1}{2}\right]} \alpha_{k} \lambda^{2 k-2} \mathcal{L}_{k}, \\
\mathcal{L}_{k} & \equiv \frac{1}{2^{k}} \delta_{\rho_{1} \cdots \rho_{k} \sigma_{1} \cdots \sigma_{k}}^{\mu_{1} \cdots \mu_{k} \nu_{1} \cdots \nu_{k}} R_{\mu_{1} \nu_{1}}^{\rho_{1} \sigma_{1}} \cdots R_{\mu_{k} \nu_{k}}^{\rho_{k} \sigma_{k}},
\end{aligned}
$$

where $[(D-1) / 2]$ denotes the integer part of $(D-1) / 2, \delta_{\rho_{1} \cdots \rho_{k} \sigma_{1} \cdots \sigma_{k}}^{\mu_{1} \cdots \mu_{k} \nu_{1} \cdots \nu_{k}}$ is the generalized anti-symmetric Kronecker delta, $\alpha_{k}$ are real dimensionless parameters and we have isolated a length (Planck) scale $\lambda$ for later convenience. This action (1) has a finite number of terms for any finite spacetime dimension. Particularly, it reduces to the Einstein action at $D=4$ with $\alpha_{0} \lambda^{-2}=-2 \Lambda$ and $\alpha_{1}=1$. The next term is the Gauss-Bonnet gravity.

On the other hand, the (closed) string effective action receives higher derivative corrections, controlled by the squared string length $\alpha^{\prime}$. These higher derivative corrections lead to ghost degrees of freedom. Since string theory itself is ghost free, it is believed that the higher derivative pathology is caused by artificial truncation and can be cured by arranging the terms properly. Therefore, Lovelock gravity should somehow can be derived from the string effective action, if string theory is the right candidate of full quantum gravity theory.

In [2], the quadratic Gauss-Bonnet term of Lovelock gravity was indeed reproduced from the first order $\alpha^{\prime}$ correction of the string effective action by field redefinitions. However, the traditional approaches seem hopeless to eliminate the higher derivatives in higher order $\alpha^{\prime}$ corrections by field redefinitions. Thus deriving Lovelock gravity from string effective action seemed not possible until recently.

Thanks to the recent remarkable progress on classifying all the $\alpha^{\prime}$ corrections done by Hohm and Zwiebach [8-10], it becomes possible to study whether Lovelock gravity matches string theory. In 1990s, Meissner and Veneziano showed that, to the first order in $\alpha^{\prime}$, when all fields only depend on time, the string effective action has an explicit $O(d, d)$ symmetry $[3,4]$. Sen proved this is true to all orders in $\alpha^{\prime}$ and for configurations independent of $m$ coordinates, the symmetry is $O(m, m)[6,7]$. This is also confirmed in ref. [5] from the perspective of $\sigma$ model expansion. It turns out that to the first order in $\alpha^{\prime}$, the $O(d, d)$ matrix can maintain the standard form in terms of $\alpha^{\prime}$ corrected fields, for time dependent configuration [3]. One can be easily convinced that this is also true for all orders in $\alpha^{\prime}$, from the derivations in [3]. Based on this assumption, Hohm and Zwiebach [8-10] showed that, for cosmological configurations, the $\alpha^{\prime}$ corrections to all orders, can be put into incredibly simple patterns. The dilaton appears trivially and only first order time derivatives need to be included. In other words, higher derivative corrections are absorbed into redefined fields by $O(d, d)$ invariant field redefinitions. In our recent work [11], we found that the 
classification of $\alpha^{\prime}$ corrections also applies to space-dependent background (domain-wall ansatz). We also presented non-singular string cosmological solutions in refs. [12, 13].

The purpose of this letter is to derive Lovelock gravity from string effective action in cosmological background. To this end, we start with the FLRW metric:

$$
d s^{2}=-d t^{2}+a(t)^{2} d x_{i} d x^{i}
$$

The Lovelock action (1) is simplified as

$$
\begin{aligned}
I_{\text {Love }}=\int d t a^{D-1} & \sum_{k=0}^{\left[\frac{D-1}{2}\right]}\left(-\frac{1}{2 k-1}\right) \alpha_{k} \lambda^{2 k-2} \times \frac{(D-1) !}{(D-2 k-1) !} H^{2 k} \\
=\int d t a^{D-1}[ & \alpha_{0} \lambda^{-2}-\alpha_{1}(D-1)(D-2) H^{2} \\
& \left.\quad-\frac{\alpha_{2} \lambda^{2}}{3}(D-1)(D-2)(D-3)(D-4) H^{4}+\cdots\right],
\end{aligned}
$$

where we modulo the spatial volume factor, and $H \equiv \dot{a}(t) / a(t)$ is the Hubble parameter. The solutions and relevant discussions of this action can be found in ref. [14].

We then turn to the string effective action corrected by all orders in $\alpha^{\prime}$ :

$$
\begin{aligned}
I_{\text {string }}=\int & d^{D} x \sqrt{-\tilde{g}} e^{-2 \phi}\left(\tilde{R}+4(\partial \phi)^{2}\right. \\
& \left.+\frac{1}{4} \alpha^{\prime}\left(\tilde{R}^{\mu \nu \rho \sigma} \tilde{R}_{\mu \nu \rho \sigma}+\ldots\right)+\alpha^{\prime 2}(\ldots)+\ldots\right),
\end{aligned}
$$

where we use "tilde" to indicate the string frame with the string metric $\tilde{g}_{\mu \nu}, \phi$ is the physical dilaton and we set Kalb-Ramond field $b_{\mu \nu}=0$ for simplicity.

Hohm and Zwiebach showed that $[9,10]$, in the FLRW ansatz, this action could be dramatically simplified as

$$
I_{\mathrm{HZ}}=\int d t e^{-\Phi}\left(-\dot{\Phi}^{2}-d \sum_{k=1}^{\infty}\left(-\alpha^{\prime}\right)^{k-1} 2^{2 k+1} c_{k} \tilde{H}^{2 k}\right),
$$

where $\Phi \equiv 2 \phi-\ln \sqrt{\operatorname{det} \tilde{g}_{i j}}$ is the $O(d, d)$ invariant dilaton. For bosonic string theory, we have $c_{1}=-\frac{1}{8}$ exactly matching Einstein gravity and $c_{2}=\frac{1}{64}$ fixing the coefficient of the Gauss-Bonnet term. $c_{k \geq 3}$ are yet unknown constants. It is worth noting that the action (5) has an explicit scale-factor duality

$$
\tilde{a}(t) \rightarrow \tilde{a}^{-1}(t), \tilde{H}(t) \rightarrow-\tilde{H}(t), \Phi(t) \rightarrow \Phi(t),
$$

which belongs to the more general $O(d, d)$ transformations.

To compare with Lovelock gravity, we need to transform the Hohm-Zwiebach action (5) to Einstein frame by

$$
\tilde{g}_{\mu \nu}=e^{-2 \omega} g_{\mu \nu}, \quad \omega \equiv-\frac{2\left(\phi-\phi_{0}\right)}{D-2}
$$


where $\phi_{0}$ is the expectation of the dilaton. However, since Lovelock gravity (3) is a pure metric theory without dilaton degree of freedom, here the string frame is same as Einstein frame. So one can simply set $\phi=\phi_{0}$ in the Hohm-Zwiebach action (5) to obtain

$$
I_{\mathrm{HZ}}=e^{-2 \phi_{0}} \int d t a^{D-1}\left[-(D-1)(D-2) H^{2}-(D-1) \sum_{k=2}^{\infty}\left(-\alpha^{\prime}\right)^{k-1} 2^{2 k+1} c_{k} H^{2 k}\right] .
$$

It is worth noting that the $O(d, d)$ symmetry (6) no longer exists after the dilaton being fixed.

At first glance, it seems this string effective action is completely different from Lovelock gravity (3), since for a specific dimension $D$, Lovelock gravity has a finite number of $\left[\frac{D+1}{2}\right]$ terms, but the string effective action has infinitely many terms for any dimension.

The point is to note that for a field theory, we are always free to make whatever regular field redefinitions which do not alter the $S$-matrix. So, if two theories can be connected by some regular field redefinitions, they are identical. For the string effective action, even at the order of $\alpha^{\prime 2}$ term, there already exist ambiguities of field redefinitions. These ambiguities simply reflect the freedom to arrange higher orders in $\alpha^{\prime}$. Therefore, to prove the equivalence of Lovelock gravity and string theory, we only need to find some regular field redefinition which transforms eq. (8) to the same pattern of eq. (3). This can be done order by order in $\alpha^{\prime}$ as follows. Substituting

$$
H^{2} \rightarrow H^{\prime 2}=H^{2}+\sum_{n=2}^{\infty}\left(-\alpha^{\prime}\right)^{n-1} A_{n} H^{2 n}
$$

into eq. (8), with the coefficients $A_{n}$,

$$
\begin{aligned}
A_{2}=-2^{5} c_{2} & \left(\frac{1}{(D-2)}+\frac{1}{6}(D-4)(D-3)\right) \\
A_{3}=-2^{7} c_{3} & \left(\frac{1}{(D-2)}-\frac{1}{24}(D-6)(D-5)(D-4)(D-3)\right) \\
& +2^{11} c_{2}^{2}\left(\frac{1}{(D-2)^{2}}+\frac{1}{6} \frac{(D-4)(D-3)}{(D-2)}\right),
\end{aligned}
$$

one can easily verify that eq. (8) fits into the same pattern of Lovelock gravity eq. (3). Then we are able to identify the relation between $c_{k}$ and $\alpha_{k}$, which will be given in eq. (15).

Note there is no constant (cosmological) term in critical string theory, so our matching starts from $H^{2}$ term. The factor $1 /(D-2)$ in denominators causes no trouble since nontrivial gravity exists in $D>2$.

Using a trick introduced by Hohm-Zwiebach in [9, 10], we can figure out the nonperturbative form of the above field redefinition by organizing the terms appropriately. We first set

$$
\delta_{k} I_{\mathrm{HZ}}=\left(-\alpha^{\prime}\right)^{k-1} e^{-2 \phi_{0}} \int d t a^{D-1} 2^{2 k+1} c_{k}(D-1) \times\left((-)^{k} \frac{1}{(k+1) !} \frac{(D-2) !}{(D-2 k-1) !}+1\right) H^{2 k},
$$


where

$$
\frac{(D-2) !}{(D-2 k-1) !}=(D-2 k) \ldots(D-3)(D-2),
$$

is naturally truncated at $k=[(D-1) / 2]$. Note the factor $(-)^{k} \frac{1}{(k+1) !}$ is used to match the coefficients of Hohm-Zwiebach action at each order. Then it is not hard to verify that the non-perturbative expression of the field redefinition (10) is

$$
\begin{aligned}
I_{\mathrm{HZ}} & \rightarrow I_{\mathrm{HZ}}^{\prime}=I_{\mathrm{HZ}}+\sum_{k=2}^{\infty} \delta_{k} I_{\mathrm{HZ}} \\
& =\int d t a^{D-1} \sum_{k=1}^{\left[\frac{D-1}{2}\right]} \beta_{k}\left(\sqrt{\alpha^{\prime}}\right)^{2 k-2} \frac{(D-1) !}{(D-2 k-1) !} H^{2 k},
\end{aligned}
$$

where

$$
\beta_{k}=-e^{-2 \phi_{0}} \frac{1}{(k+1) !} 2^{2 k+1} c_{k} .
$$

We redefined $c_{1}=\frac{1}{4}$ to replace its original value $-\frac{1}{8}$ to make the expressions simpler. Comparing with Lovelock gravity (3), it is ready to find

$$
\alpha_{k}=\frac{2 k-1}{(k+1) !} 2^{2 k+1} c_{k}
$$

after identifying $\lambda \equiv \sqrt{\alpha^{\prime}}$. The coupling constants $\alpha_{k}$ in Lovelock gravity were arbitrary. However, we now see that as an effective theory of string theory, these couplings are fixed by the corresponding coefficients in the string effective action.

One enlightening observation from our derivation is that, when a proper field redefinition is used, infinitely many $\alpha^{\prime}$ corrections are replaced by finite terms for a specific dimension, say $D=26$. If this is also true when the dilaton is turned on, we are able actually to get a closed expression for the string spacetime action. Therefore, we may fix the non-perturbative string theory through perturbative results.

Moreover, we wish to address a closely related subject, the quasi-topological gravity, which was first proposed by Myers and Robinson [17] under a specialized black hole ansatz. In refs. [18-20], the quasi-topological gravity was extended to FLRW background. As we have mentioned, Lovelock theorem asserts that there is no alternative generalization of Einstein's gravity, which still possesses the second-order equations of motion. Myers and Robinson adopted a different way. They first select all possible curvature-cubed interactions. Then, all these curvature-cubed interactions are combined together with undetermined coefficients. For some special metric ansatz, it is possible to fix the relations between coefficients to guarantee the equations of motion to be second-order. Finally, using the interactions with fixed coefficients, the ghost-free generalised action for that special metric ansatz was obtained. Therefore, the quasi-topological gravity could allow cubic terms active in five dimensions, but only for some preselected special background, as opposed to cubic Lovelock gravity only exists in seven dimensions for general background. In addition to quasi-topological gravity, there are some other higher-curvature generalizations of gravity $[21,22]$. 
Based on our derivation, it is possible to ask whether these different kind of generalizations of gravity could be related to Hohm-Zwiebach action by appropriate field redefinitions. The answer is tricky. It should be noted there are two sorts of coefficients in string effective action, namely, ambiguous and unambiguous coefficients. The unambiguous coefficients are independent of field redefinitions, while the ambiguous coefficients are altered by field redefinitions. The observables of string theory, say S-matrix, are controlled by unambiguous coefficients. This is why the field redefinitions do not affect physical results. In Hohm and Zwiebach's work, a series of field redefinitions were used to set all ambiguous coefficients vanishing altogether, and only the unambiguous coefficients $2^{2 k+1} c_{k}$ are left to dramatically simplify the action. To keep the coefficients $2^{2 k+1} c_{k}$ invariant, the $O(d, d)$ invariant field redefinitions, namely $\delta_{k} I_{\mathrm{HZ}}$, only can provide following terms to modify ambiguous coefficients in the low energy effective action:

$$
\left[\sum_{i=0}^{2 k-1} a_{i} D^{i}\right]\left(\sqrt{\alpha^{\prime}}\right)^{2 k-2} H^{2 k}
$$

where $a_{i}$ are arbitrary constants and $D^{i}$ denotes an $i$-th power of the spacetime dimension $D$. It is worth noting that $a_{i}$ do not depend on the spacetime dimension. However, a curvature-cubed term of the quasi-topological gravity takes a form:

$$
\left[\sum_{i=0}^{5} \frac{a_{i}}{(2 D-3)} D^{i}\right] \mu_{3} H^{6}
$$

where $\mu_{3}$ is an arbitrary dimensional parameter with dimension (Length) ${ }^{4}$, and it does not depend on the spacetime dimension. We can see that the unambiguous coefficients are absent in (17) and there is no way to find an appropriate $O(d, d)$ invariant field redefinition to cancel the $2 D-3$ denominator in (17) to match eq. (16). Therefore, we cannot reach the quasi-topological gravity by field redefinitions from Hohm-Zwiebach action.

Moreover, we need to note that generalizing Hohm-Zwiebach action to a general background is not available yet, because the $O(d, d)$ symmetry constraints the ansatz of background. We believe this problem could be solved by double field theory.

In summary, we showed that Lovelock gravity can be derived from Hohm-Zwiebach action via an appropriate field redefinition. The couplings of Lovelock gravity are fixed by the coefficients of string effective action. Although our discussion only applies to cosmological background, it does provide a very strong evidence for string theory. Generalizing our discussion to situations that rely on two or more coordinates are very desirable. To this end, we need to extend Hohm-Zwiebach action to depending on more than just one variable. The first nontrivial step on this track recently has been done in $[15,16]$.

\section{Acknowledgments}

This work is supported in part by the NSFC (Grant No. 11875196, 11375121, 11005016 and 11947225). 
Open Access. This article is distributed under the terms of the Creative Commons Attribution License (CC-BY 4.0), which permits any use, distribution and reproduction in any medium, provided the original author(s) and source are credited.

\section{References}

[1] D. Lovelock, The Einstein tensor and its generalizations, J. Math. Phys. 12 (1971) 498 [INSPIRE].

[2] B. Zwiebach, Curvature Squared Terms and String Theories, Phys. Lett. B 156 (1985) 315 [INSPIRE].

[3] K.A. Meissner, Symmetries of higher order string gravity actions, Phys. Lett. B 392 (1997) 298 [hep-th/9610131] [INSPIRE].

[4] G. Veneziano, Scale factor duality for classical and quantum strings, Phys. Lett. B 265 (1991) 287 [INSPIRE].

[5] K.A. Meissner and G. Veneziano, Symmetries of cosmological superstring vacua, Phys. Lett. B 267 (1991) 33 [INSPIRE].

[6] A. Sen, $O(d) x O(d)$ symmetry of the space of cosmological solutions in string theory, scale factor duality and two-dimensional black holes, Phys. Lett. B 271 (1991) 295 [INSPIRE].

[7] A. Sen, Twisted black p-brane solutions in string theory, Phys. Lett. B 274 (1992) 34 [hep-th/9108011] [INSPIRE].

[8] O. Hohm and B. Zwiebach, T-duality Constraints on Higher Derivatives Revisited, JHEP 04 (2016) 101 [arXiv: 1510.00005] [INSPIRE].

[9] O. Hohm and B. Zwiebach, Non-perturbative de Sitter vacua via $\alpha^{\prime}$ corrections, Int. J. Mod. Phys. D 28 (2019) 1943002 [arXiv:1905.06583] [INSPIRE].

[10] O. Hohm and B. Zwiebach, Duality invariant cosmology to all orders in $\alpha$ ', Phys. Rev. D 100 (2019) 126011 [arXiv:1905.06963] [INSPIRE].

[11] P. Wang, H. Wu and H. Yang, Are nonperturbative AdS vacua possible in bosonic string theory?, Phys. Rev. D 100 (2019) 046016 [arXiv:1906.09650] [InSPIRE].

[12] P. Wang, H. Wu, H. Yang and S. Ying, Non-singular string cosmology via $\alpha^{\prime}$ corrections, JHEP 10 (2019) 263 [arXiv:1909.00830] [INSPIRE].

[13] P. Wang, H. Wu, H. Yang and S. Ying, Construct $\alpha^{\prime}$ corrected or loop corrected solutions without curvature singularities, JHEP 01 (2020) 164 [arXiv:1910.05808] [INSPIRE].

[14] N. Deruelle and L. Farina-Busto, The Lovelock Gravitational Field Equations in Cosmology, Phys. Rev. D 41 (1990) 3696 [InSPIRE].

[15] C. Eloy, O. Hohm and H. Samtleben, Green-Schwarz Mechanism for String Dualities, Phys. Rev. Lett. 124 (2020) 091601 [arXiv: 1912.01700] [InSPIRE].

[16] C. Eloy, O. Hohm and H. Samtleben, Duality Invariance and Higher Derivatives, Phys. Rev. D 101 (2020) 126018 [arXiv:2004.13140] [INSPIRE].

[17] R.C. Myers and B. Robinson, Black Holes in Quasi-topological Gravity, JHEP 08 (2010) 067 [arXiv: 1003.5357] [INSPIRE].

[18] M.H. Dehghani, A. Sheykhi and R. Dehghani, Thermodynamics of Quasi-Topological Cosmology, Phys. Lett. B $\mathbf{7 2 4}$ (2013) 11 [arXiv:1306.4510] [InSPIRE]. 
[19] A. Cisterna, N. Grandi and J. Oliva, On four-dimensional Einsteinian gravity, quasitopological gravity, cosmology and black holes, Phys. Lett. B 805 (2020) 135435 [arXiv: 1811.06523] [INSPIRE].

[20] G. Arciniega, J.D. Edelstein and L.G. Jaime, Towards geometric inflation: the cubic case, Phys. Lett. B 802 (2020) 135272 [arXiv:1810.08166] [InSPIRE].

[21] G. Arciniega, P. Bueno, P.A. Cano, J.D. Edelstein, R.A. Hennigar and L.G. Jaime, Geometric Inflation, Phys. Lett. B 802 (2020) 135242 [arXiv:1812.11187] [InSPIRE].

[22] P.A. Cano, K. Fransen and T. Hertog, Novel higher-curvature variations of $R^{2}$ inflation, arXiv:2011.13933 [INSPIRE]. 